\title{
Meeting Patient Expectations or Achieving a Minimum Clinically Important Difference: Predictors of Satisfaction among Lumbar Fusion Patients
}

\author{
Elliot D. K. Cha, Conor P. Lynch, Caroline N. Jadczak, Shruthi Mohan, Cara E. Geoghegan, Kern Singh \\ Department of Orthopaedic Surgery, Rush University Medical Center, Chicago, IL, USA
}

\begin{abstract}
Study Design: Retrospective cohort.
Purpose: To investigate the impact of meeting a patient's preoperative expectations for back or leg pain or the achievement of minimum clinically important difference (MCID) on patient satisfaction following lumbar fusion.

Overview of Literature: Few studies have compared if MCID achievement or meeting preoperative expectations for pain reduction affects patient satisfaction.

Methods: A surgical database was reviewed for eligible patients who underwent lumbar fusion. Patient satisfaction and Visual Analog Scale (VAS) for back and leg pain were the outcomes of interest. Meeting expectations was calculated as a difference of $\leq 0$ between preoperative expectations and postoperative VAS scores. MCID achievement was calculated by comparing changes in VAS scores with established values. Meeting preoperative expectations or MCID achievement as predictors of patient satisfaction was evaluated using regression analysis.

Results: A total of 134 patients were included in this study. Patients demonstrated significant improvements in VAS back and VAS leg $(p<0.001)$. At 1 year, $56.4 \%$ of patients had their VAS back expectations met compared with $59.5 \%$ for VAS leg. Similarly, at 1 year, $77.3 \%$ and $71.3 \%$ of patients achieved MCID for VAS back and leg, respectively. Meeting expectations for VAS back was significantly associated with patient satisfaction at all postoperative timepoints; however, MCID achievement only demonstrated a significant association with patient satisfaction at 6 and 12 weeks (all, $p \leq 0.024$ ). Meeting VAS leg expectations and MCID achievement both demonstrated a significant association with patient satisfaction at all postoperative timepoints (all, $p \leq 0.02$ ). No differences between MCID achievement and meeting expectations as predictors of satisfaction were noted.

Conclusions: The majority of patients achieved MCID and had their back and leg pain expectations met by 1 year. Both measures were significant predictors of patient satisfaction and suggest that MCID achievement may act as a suitable substitute for patient satisfaction.
\end{abstract}

Keywords: Patient reported outcome measures; Minimal clinically important difference; Spinal fusion

Received Apr 8, 2021; Revised Jun 2, 2021; Accepted Jun 3, 2021

Corresponding author: Kern Singh

Department of Orthopaedic Surgery, Rush University Medical Center, 1611 W. Harrison St, Suite \#300, Chicago, IL 60612, USA

Tel: +1-312-432-2373, Fax: +1-708-409-5179, E-mail: kern.singh@rushortho.com 


\section{Introduction}

Debilitating lower back pain is a large source of patient dissatisfaction and is one of the most important factors for patients when deciding to opt for lumbar fusion surgery $[1,2]$. Physicians can gauge postoperative improvement in back and leg pain through the use of validated patientreported outcome measures (PROMs), such as the Visual Analog Scale (VAS) [3]. Although VAS may reach significantly lower values than the preoperative score, the metric offers no information regarding the associated level of patient satisfaction. Studies have reported a strong association between satisfaction and postoperative outcomes [4], although they have noted that this can be influenced by preoperative expectations.

Owing to the significance placed on pain improvement, patients may tend to set high expectations, which, if not met, can result in poorer reported outcomes and higher dissatisfaction $[5,6]$. Several investigators have reported that higher preoperative expectations can lead to better outcomes and higher satisfaction $[7,8]$, whereas other studies suggest the opposite, wherein a higher expectation results in higher dissatisfaction and worse outcomes among lumbar spine patients $[9,10]$. Variability in the current literature makes it challenging for physicians to accurately evaluate whether a patient is satisfied owing to meeting preoperative expectations and/or successful alleviation of symptomatic pain following surgical intervention.

Instead, a more reliable metric that considers patient perception is the minimum clinically important difference (MCID). MCID is defined as the smallest appreciable difference in scores that a patient perceives as beneficial [11] and removes the burden of comparing multiple preoperative and postoperative PROM scores that may or may not be indicative of true satisfaction and, instead, introduces score thresholds. Several studies have demonstrated that MCID is a strong predictor of patient satisfaction and functional clinical improvement among lumbar spine patients [12-14].

Although physicians may have various options, as far as metrics is concerned, to evaluate patient satisfaction, few studies have distinguished whether meeting expectations or overall improvement in the quality-of-life measures is more reliable and accurate in predicting patient satisfaction. Moreover, the high variability regarding a patient's preoperative expectations makes predicting the extent of satisfaction difficult. Therefore, our study aims to answer this question by evaluating whether meeting expectations or achieving MCID is a stronger predictor of patient satisfaction among patients undergoing lumbar fusion.

\section{Materials and Methods}

\section{Patient identification}

Eligible lumbar procedures from October 2016 to September 2020 were identified through a retrospective review of a prospective spine surgery database. Patients who underwent a primary, single, or multilevel lumbar fusion procedure with posterior instrumentation were included in this study. Exclusion criteria were set as revision procedures or those indicated for infection, trauma, or malignancy. Furthermore, patients were excluded for failing to complete a preoperative pain expectations questionnaire. A total of 217 patients were identified for this study, with 134 remaining after inclusion and exclusion criteria were applied (Fig. 1). Following institutional guidelines, Rush University Medical Center Institutional Review Board approval (IRB approval no., ORA \#14051301) and written patient informed consent were obtained before the commencement of this study.

\section{Surgical technique}

All patients underwent a primary, single, or multilevel anterior, lateral, or transforaminal lumbar interbody fusion (TLIF) with posterior instrumentation. A single spine surgeon performed all lumbar fusion procedures.

\section{Data acquisition}

Patient demographics or perioperative characteristics

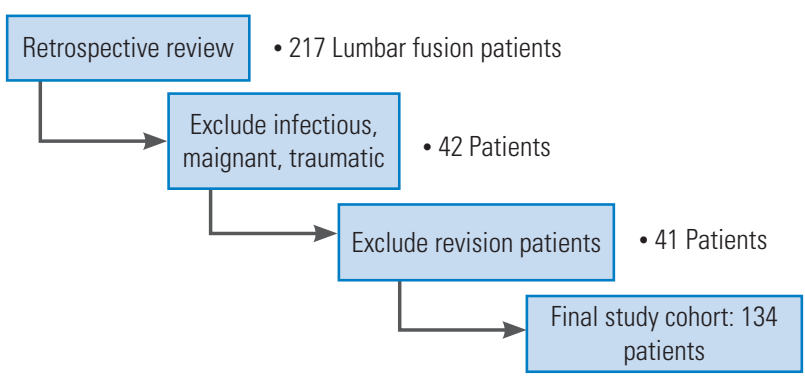

Fig. 1. Inclusion and exclusion of patients. 
were collected through a retrospective chart review. The demographics included age, sex, body mass index, smoker status, diabetic status, and insurance collected. The preoperative physical classification was collected as the American Society of Anesthesiologists score and patient comorbidity burden as the Charlson comorbidity index. The proportion of patients experiencing a high level of back or leg pain was preoperatively evaluated using VAS. Perioperative information was collected as spinal pathology, the number of levels fused, operative levels, operative duration (from skin incision to skin closure), estimated blood loss, length of postoperative hospital stay, day of discharge, and postoperative complications.

Patient satisfaction was the primary outcome of interest, measured on a continuous scale from 0 to 10 , with 10 being the most satisfied and 0 being the least satisfied. Additionally, VAS back and VAS leg were collected at the preoperative timepoint and at the 6-week, 12-week, 6-month, and 1-year postoperative timepoints. MCID achievement was evaluated by comparing the difference in postoperative from preoperative VAS back or VAS scores with established values: 1.2 (VAS back) [13] and 1.6 (VAS leg) [13]. Meeting patient expectations was defined as a difference of $\leq 0$ between preoperative and postoperative VAS scores.

\section{Statistical analysis}

Descriptive statistics were performed for baseline characteristics and all outcomes of interest. Changes in postoperative VAS back and leg scores from baseline values were evaluated using the paired Student $t$-test. Significant baseline covariates associated with either MCID achievement or meeting preoperative expectations were evaluated using Poisson regression with robust error variance. The correlation between outcomes and satisfaction scores was evaluated using Pearson coefficient, and the strength of correlation was categorized as weak $(|r|<0.3)$, moderate $(0.3 \leq|r|<0.5)$, or strong $(|r| \geq 0.5)$. Simple linear regression was performed to determine the effect of either meeting patient expectations or MCID achievement on patient satisfaction. The comparison of meeting expectations or MCID achievement as predictors of patient satisfaction was evaluated using the post-estimation (suest) test. A value of $a=0.050$ was set to reject the null hypothesis. All statistical tests were performed using Stata ver. 16.1 (Stata Corp., College Station, TX, USA).

\section{Results}

The study cohort of 134 patients had a mean age of 54.6 years, $59.7 \%$ were male, and $51.5 \%$ were obese (body mass index $\geq 30.0 \mathrm{~kg} / \mathrm{m}^{2}$ ). The majority of patients were non-smokers and non-diabetic (both, 88.8\%) and had an American Society of Anesthesiologists score of $\leq 2$ (77.7\%) and a higher Charlson comorbidity index value (54.3\%) (Table 1). The majority of patients had a spinal pathology of degenerative spondylolisthesis (56.5\%). Lumbar fusions

Table 1. Patient demographics ( $\mathrm{N}=134)$

\begin{tabular}{|c|c|}
\hline Characteristic & Value \\
\hline Age (yr) & $54.6 \pm 10.2$ \\
\hline \multicolumn{2}{|l|}{ Gender } \\
\hline Female & $40.3(54)$ \\
\hline Male & $59.7(80)$ \\
\hline \multicolumn{2}{|l|}{ Body mass index $\left(\mathrm{kg} / \mathrm{m}^{2}\right)$} \\
\hline$<30.0$ & $48.5(64)$ \\
\hline$\geq 30.0$ & $41.5(68)$ \\
\hline \multicolumn{2}{|l|}{ Smoking status } \\
\hline Non-smoker & $88.8(119)$ \\
\hline Smoker & $11.2(15)$ \\
\hline \multicolumn{2}{|l|}{ Diabetes } \\
\hline Non-diabetic & $88.8(119)$ \\
\hline Diabetic & $11.2(15)$ \\
\hline \multicolumn{2}{|c|}{ American Society of Anesthesiologists score } \\
\hline$\leq 2$ & $77.7(101)$ \\
\hline$>2$ & $22.3(29)$ \\
\hline \multicolumn{2}{|c|}{ Charlson comorbidity index score } \\
\hline$<1$ & $45.7(48)$ \\
\hline$\geq 1$ & $54.3(57)$ \\
\hline \multicolumn{2}{|l|}{ VAS back } \\
\hline$<7$ & $56.1(74)$ \\
\hline$\geq 7$ & $43.9(58)$ \\
\hline \multicolumn{2}{|l|}{ VAS leg } \\
\hline$<7$ & $62.4(83)$ \\
\hline$\geq 7$ & $37.6(50)$ \\
\hline \multicolumn{2}{|l|}{ Insurance } \\
\hline Medicare/Medicaid & $2.2(3)$ \\
\hline Workers' compensation & $23.1(31)$ \\
\hline Private & $74.7(100)$ \\
\hline
\end{tabular}

Values are presented as mean \pm standard deviation or \% (number). VAS, Visual Analog Scale. 
were typically performed on a single level (85.1\%) at the L4-5 level (40.0\%). The operative duration was $141.5 \mathrm{~min}$ utes on average, with a mean estimated blood loss of 47.6 $\mathrm{mL}$. Patients had a length of postoperative hospital stay of 27.9 hours, with the majority discharged on postoperative day $1(56.3 \%)$. A summary of perioperative characteristics is presented in Table 2. The bivariate analysis identified the high preoperative leg pain as a significant covariate for meeting expectations and MCID achievement for the VAS leg. The number of operative levels and high preoperative back pain were significant covariates for MCID achievement for VAS back. The comorbidity burden was a significant covariate for VAS leg MCID achievement $(p<0.05)$. A total of two postoperative complications were recorded, with two individuals with urinary retention requiring straight catheterization, although spontaneously voided before discharge. The complications were not significant covariates $(p>0.05)$.

The study cohort reported a mean satisfaction score of $6.6 \pm 3.1,6.6 \pm 3.5,5.8 \pm 3.4$, and $6.7 \pm 3.6$ at 6 weeks, 12

Table 2. Perioperative characteristics ( $N=134)$

\begin{tabular}{lc} 
Characteristic & Value \\
Spinal pathology & \\
\hline Degenerative spondylolisthesis & $56.5(74)$ \\
\hline Isthmic spondylolisthesis & $26.7(35)$ \\
\hline Recurrent herniated nucleus pulposus & $9.0(12)$ \\
\hline No. of fusion levels & \\
\hline Single & $85.1(114)$ \\
\hline Multilevel & $14.9(20)$ \\
\hline Fusion level & \\
\hline L3-4 & $6.0(8)$ \\
\hline L3-5 & $6.0(8)$ \\
\hline L4-5 & $40.3(54)$ \\
\hline L5-S1 & $38.1(51)$ \\
\hline Operative time (min) & $141.5 \pm 49.4$ \\
\hline Estimated blood loss (mL) & $47.6 \pm 25.1$ \\
\hline Length of stay (hr) & $27.9 \pm 20.7$ \\
\hline Day of discharge & \\
\hline P0D0 & $24.6(31)$ \\
\hline P0D1 & $56.3(71)$ \\
\hline P0D2 & $15.1(19)$ \\
\hline P0D3 & $4.0(5)$ \\
\hline
\end{tabular}

Values are presented as \% (number) or mean \pm standard deviation. POD, postoperative day. weeks, 6 months, and 1 year for VAS back, respectively. The satisfaction scores for the VAS leg were 7.2 \pm 3.2 , $7.1 \pm 3.2,6.8 \pm 3.7$, and $6.9 \pm 3.6$ at 6 weeks, 12 weeks, 6 months, and 1 year, respectively. The mean preoperative VAS back was $5.9 \pm 2.5$ and demonstrated significant improvements from baseline values at the 6-week, 12week, 6-month, and 1-year timepoints (all, $p<0.001$ ). The mean preoperative VAS leg was $5.3 \pm 29$ and significantly improved at the 6-week through 1-year timepoints (all, $p<0.001$ ) (Table 3). Both VAS back and VAS leg demonstrated significantly negative and strong correlations with satisfaction scores at all postoperative timepoints $(|r| \geq 0.5$, $p<0.001)$. The majority of patients $(>50.0 \%)$ achieved an MCID for both VAS back and VAS leg at all timepoints. Additionally, $>70 \%$ of patients achieved an MCID for VAS back or VAS leg by the 1-year timepoint. The majority of patients had their pain expectations met for VAS back only at the 1-year timepoint, whereas the majority of patients had their expectations for VAS leg met at the 6-week, 6-month, and 1-year timepoints (Table 3).

Meeting expectations for VAS back demonstrated significant effects on VAS back satisfaction scores at the 6-week, 12-week, 6-month, and 1-year timepoints (all, $p \leq 0.012$ ). A similar finding was also demonstrated for the VAS leg, with meeting expectations being a significant effector of satisfaction at the 6-week through 1-year timepoints (all, $p<0.01$ ) (Table 4). MCID achievement for VAS back was only significantly associated with satisfaction scores at the 6- and 12-week timepoints (both, $p \leq 0.024$ ). MCID achievement for the VAS leg was significantly associated with satisfaction scores from the 6-week through 1 -year timepoints (all, $p<0.05$ ). The comparison of meeting expectations and MCID achievement as effectors of patient satisfaction demonstrated significant differences only at the 6-week timepoint for the VAS leg $(p=0.013)$ (Table 4).

\section{Discussion}

Value-based healthcare has placed increased importance on patient-reported outcomes and satisfaction to evaluate postoperative improvement and quality of life. Although improvements regarding pain, disability, and physical function may follow a more predictable path, patient satisfaction may be influenced by expectations regarding the resolution of pain or disability. In contrast, the establishment of an objective MCID has been demonstrated to re- 
Table 3. Postoperative improvement and MCID achievement

\begin{tabular}{|c|c|c|c|c|c|}
\hline Variable & PROM & $p$-value ${ }^{a)}$ & Satisfaction & Met expectations & Achieved MCID \\
\hline \multicolumn{6}{|l|}{ VAS back } \\
\hline Preoperative & $5.9 \pm 2.5(132)$ & - & - & - & - \\
\hline 6-wk & $3.6 \pm 2.5(106)$ & $<0.001$ & $6.6 \pm 3.1$ & $40.0(36)$ & $58.5(62)$ \\
\hline $12-w k$ & $3.6 \pm 2.6(96)$ & $<0.001$ & $6.6 \pm 3.5$ & $47.5(38)$ & $60.4(58)$ \\
\hline 6-mo & $3.5 \pm 2.7(82)$ & $<0.001$ & $5.8 \pm 3.4$ & $35.2(25)$ & $67.1(55)$ \\
\hline $1-y r$ & $3.2 \pm 2.8(32)$ & $<0.001$ & $6.7 \pm 3.6$ & $52.7(29)$ & $62.5(20)$ \\
\hline Overall & - & & - & - & $77.3(92)$ \\
\hline \multicolumn{6}{|l|}{ VAS leg } \\
\hline Preoperative & $5.3 \pm 2.9(133)$ & - & - & - & - \\
\hline 6-wk & $3.0 \pm 2.9(106)$ & $<0.001$ & $7.2 \pm 3.2$ & $53.3(48)$ & $52.8(56)$ \\
\hline $12-w k$ & $2.6 \pm 2.9(97)$ & $<0.001$ & $7.1 \pm 3.2$ & $48.7(39)$ & $61.9(60)$ \\
\hline 6-mo & $2.7 \pm 3.0(83)$ & $<0.001$ & $6.8 \pm 3.7$ & $52.1(37)$ & $57.8(48)$ \\
\hline $1-y r$ & $3.2 \pm 3.2(32)$ & $<0.001$ & $6.9 \pm 3.6$ & $54.5(30)$ & $53.1(17)$ \\
\hline Overall & - & & - & - & $71.3(82)$ \\
\hline
\end{tabular}

Values are presented as mean $\pm S D$ (number), mean $\pm S D$, or \% (number), unless otherwise stated. Boldface indicates statistical significance.

MCID, minimum clinically important difference; PROM, patient-reported outcome measure; VAS, Visual Analog Scale; SD, standard deviation.

${ }^{\text {al }}$ Calculated using a paired $t$-test to compare preoperative to postoperative values.

Table 4. Predictors of satisfaction

\begin{tabular}{|c|c|c|c|c|c|c|c|}
\hline \multirow{2}{*}{ Variable } & \multicolumn{3}{|c|}{ Meeting expectations } & \multicolumn{3}{|c|}{ Achieving MCID } & \multirow{2}{*}{$p$-value ${ }^{b)}$} \\
\hline & Coefficient & $R^{2}$ & $p$-value ${ }^{a)}$ & Coefficient & $R^{2}$ & $p$-value ${ }^{a)}$ & \\
\hline \multicolumn{8}{|l|}{ VAS back } \\
\hline 6-wk & 1.94 & 0.097 & $<0.001$ & 2.71 & 0.190 & 0.003 & 0.320 \\
\hline $12-w k$ & 1.69 & 0.077 & 0.012 & 1.60 & 0.068 & 0.024 & 0.917 \\
\hline 6-mo & 2.29 & 0.105 & 0.006 & 1.81 & 0.057 & 0.064 & 0.697 \\
\hline $1-y r$ & 3.82 & 0.292 & $<0.001$ & 2.36 & 0.123 & 0.085 & 0.339 \\
\hline \multicolumn{8}{|l|}{ VAS leg } \\
\hline 6-wk & 3.90 & 0.371 & $<0.001$ & 1.90 & 0.091 & 0.004 & 0.013 \\
\hline $12-w k$ & 3.78 & 0.345 & $<0.001$ & 3.62 & 0.284 & $<0.001$ & 0.865 \\
\hline 6-mo & 2.46 & 0.113 & 0.004 & 1.93 & 0.066 & 0.044 & 0.620 \\
\hline $1-y r$ & 4.83 & 0.447 & $<0.001$ & 3.73 & 0.261 & 0.009 & 0.405 \\
\hline
\end{tabular}

Boldface indicates statistical significance.

MCID, minimum clinically important difference; VAS, Visual Analog Scale.

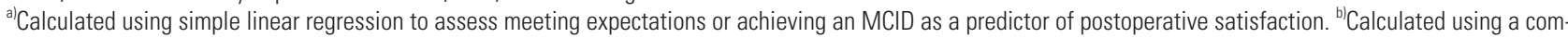
parison of coefficients to determine differences in effect size between meeting expectations and achieving MCID as predictors of satisfaction.

late satisfaction with postoperative improvements [12-15]. Few studies have compared the strength of association between the subjective "meeting of expectations" or MCID achievement with patient satisfaction. The current study evaluates not only the respective associations with patient satisfaction but also if one metric may be better suited to predict satisfaction scores.

Lumbar fusion is well established as an effective treatment for the relief of both back and leg pain. Our study made a similar observation along with establishing a significant negative correlation with satisfaction scores. Yoo et al. [4] reported a similar significant and negative cor- 
relation with not only VAS back and VAS leg but also with Oswestry Disability Index (ODI). Other investigators have also reported similar relationships, although in the context of their roles as predictors of satisfaction. For instance, Lim et al. [16] demonstrated that among patients with TLIF, the relief of leg pain was associated with patient satisfaction, and individuals with a higher preoperative score had a higher likelihood of being satisfied with the surgery. Interestingly, patients with a higher back pain score were associated with a higher dissatisfaction score, which may be mitigated if patients presented with more severe preoperative leg pain [17]. More robust studies have also highlighted the significance of back pain predominance in decreasing the likelihood of patient satisfaction [18]. Other prior studies on TLIF also explored the implication of preoperative leg or back pain predominance on outcome improvement and reported no significant difference in the rates of MCID achievement for VAS back ( $80.9 \%$ versus $76.3 \%)$, VAS leg (82.4\% versus $92.1 \%)$, and ODI (61.8\% versus $68.4 \%)$ at the 1-year timepoint [19]. Although our current study did not classify which source of pain predominated, back or leg, the patient cohort reported a similar patient satisfaction score at each postoperative timepoint along with similar rates of MCID achievement and meeting expectations for VAS back and VAS leg at 1 year. Our results do support past studies; however, inconsistent associations between back and leg pain resolution and patient satisfaction highlight the subjective nature of pain itself and support the requirement for a more consistent and objective predictor.

Determining whether baseline expectations for the resolution of back or leg pain were "met" could act as a predictor of satisfaction. The current study established that meeting expectations for VAS back and/or VAS leg were significant predictors of patient satisfaction at all postoperative timepoints. Although Yoo et al. [4] established that preoperative expectations were not correlated with satisfaction scores, other investigators demonstrated that meeting expected postoperative pain scores acted as a significant predictor, a result that is consistent with the result of our study $[9,20,21]$. More specifically, both Soroceanu et al. [9] and Toyone et al. [20] established that odds of patients being satisfied with postsurgical outcomes were increased with the fulfillment of expectations regarding pain relief ( $p=0.008$ and $p=0.016$, respectively), whereas Mannion et al. [21] established that meeting expectations, as compared with the actual improvement of outcomes, governs more positive outcomes. Although our study and others demonstrate that meeting expectations can predict satisfaction with the surgery, a patient's assessment of what they perceive as an acceptable improvement is ultimately formed on their own regardless of preoperative counseling. Further confounding the matter are factors, such as younger age, male, greater disability, and worse mental and physical health scores, which have been associated with greater expectations before surgery $[10,22]$. This suggests that although a significant predictor of satisfaction, meeting a patient's preoperative expectations could be highly variable, leading to inconsistent evaluation of postoperative outcomes.

MCID achievement may be a better suited metric to predict patient satisfaction. The patients in our cohort that demonstrated significant improvements and achieved an MCID for both VAS back (1.2) [13] and VAS leg (1.6) [13] significantly predicted satisfaction scores. This is to be expected since previous studies calculated and established the threshold values for both cervical and lumbar procedures using satisfaction indices as anchors [12,13,15,23], which delineate between a significant or non-significant change in outcomes as perceived by the patient. Beyond the establishment of an MCID value, few other studies have reported a significant relationship with satisfaction scores. Carragee and Cheng [14] used MCID achievement as a way to define the "success" of a spinal procedure and reported a strong association with satisfaction for up to 2 years. Comparatively, the current study only established MCID achievement as a predictor of satisfaction for leg pain up to the 1-year timepoint and only to the 12-week timepoint for VAS back. Although VAS leg largely replicates the findings by Carragee and Cheng [14], differences in the ability of VAS back to predict satisfaction scores may be attributed to the indication and effect of the procedure itself. The main indication for lumbar surgery is the frequently intractable radicular leg pain, which is typically resolved following the procedure [24]. However, our results, along with past studies, indicate that MCID achievement remains an equivalent and, in one instance, a better predictor of patient satisfaction than "meeting" patient expectations for pain resolution.

Establishing the equivalence of these two metrics as predictors of patient satisfaction highlights the strength of this study. Few studies have conducted a similar comparison and our results support the use of MCID instead of meeting expectations as a consistent and less variable 
measurement to predict postoperative satisfaction. Despite appropriate preoperative counseling, having high expectations can ultimately cause a patient to be disappointed with their outcomes and, in turn, may negatively influence patient-reported outcomes. The use of MCID rather than meeting expectations will thereby allow surgeons to appropriately gauge and predict the level of patient satisfaction without having to account for potential confounders that may inflate or deflate expectations and thereby satisfaction scores.

This study has several limitations that should be addressed. First, the potential for bias is one of the most apparent limitations since the majority of the reported data was based on self-reported questionnaires and may be prone to recall or responder bias. Second, the inclusion of several different lumbar fusion procedures could alter postoperative outcomes or even satisfaction scores. Additionally, the procedures were performed by a single spine surgeon at a single institution, which included outpatient facilities. Together, this could introduce a bias toward one particular demographic and could result in more favorable satisfaction scores depending on the familiarity of the patient with the surgeon and surgical center. Although, clinically, not an unfavorable bias, a multi-provider or multi-institutional approach should be used in future studies to broaden the generalizability of the results. Moreover, the retrospective nature of the study may introduce some level of selection bias and will limit our ability to perform a randomized comparison of predictors of patient satisfaction. Lastly, the exclusion of patients with incomplete satisfaction surveys may introduce a selection bias for patients who have more favorable outcomes. Prior studies have alluded to non-compliant patients having less favorable outcomes [25,26]; however, other studies have also reported no difference [27]. Clinicians should be mindful that lower compliance may artificially inflate satisfaction scores and may need to closely follow the progress of patients who may not complete questionnaires.

\section{Conclusions}

Patients undergoing lumbar fusion procedures achieved a significant improvement in both back and leg pain at 1 year postoperatively. Additionally, the majority of individuals were able to achieve an MCID for both leg and back pain at 1 year, although the same was not observed for meeting the patient's preoperative expectations for the resolution of pain. Meeting expectations and MCID achievement were associated with patient satisfaction, and direct comparisons demonstrated no significant difference in their effect. These results suggest that MCID achievement may be a suitable and less variable measure to rely on to determine or predict patient satisfaction following lumbar fusion surgery.

\section{Conflict of Interest}

No potential conflict of interest relevant to this article was reported.

\section{Author Contributions}

Conception and design: EDKC, CPL, KS; analysis of data: EDKC, CPL; drafting manuscript: EDKC, CPL, CNJ; critical revision: EDKC, CPL, CNJ, SM, CEG, KS; data acquisition: CNJ, SM, CEG, KS; administrative support: CNJ, SM, CEG; and supervision: KS.

\section{References}

1. Bono CM, Harris MB, Warholic N, et al. Pain intensity and patients' acceptance of surgical complication risks with lumbar fusion. Spine (Phila Pa 1976) 2013;38:140-7.

2. Bederman SS, Mahomed NN, Kreder HJ, McIsaac WJ, Coyte PC, Wright JG. In the eye of the beholder: preferences of patients, family physicians, and surgeons for lumbar spinal surgery. Spine (Phila $\mathrm{Pa}$ 1976) 2010;35:108-15.

3. Zanoli G, Stromqvist B, Jonsson B. Visual analog scales for interpretation of back and leg pain intensity in patients operated for degenerative lumbar spine disorders. Spine (Phila Pa 1976) 2001;26:2375-80.

4. Yoo JS, Patel DV, Mayo BC, et al. Postoperative satisfaction following lumbar spinal fusion surgery: patient expectation versus actuality. J Neurosurg Spine 2019;31:676-82.

5. Jones KR, Burney RE, Christy B. Patient expectations for surgery: are they being met? Jt Comm J Qual Improv 2000;26:349-60.

6. Judge A, Cooper C, Arden NK, et al. Pre-operative expectation predicts 12 -month post-operative outcome among patients undergoing primary total hip replacement in European orthopaedic centres. Os- 
teoarthritis Cartilage 2011;19:659-67.

7. Krauss P, Sonnleitner C, Reinartz F, Meyer B, Meyer HS. Patient-reported expectations, outcome and satisfaction in thoracic and lumbar spine stabilization surgery: a prospective study. Surgeries 2020;1:63-76.

8. Saban KL, Penckofer SM. Patient expectations of quality of life following lumbar spinal surgery. J Neurosci Nurs 2007;39:180-9.

9. Soroceanu A, Ching A, Abdu W, McGuire K. Relationship between preoperative expectations, satisfaction, and functional outcomes in patients undergoing lumbar and cervical spine surgery: a multicenter study. Spine (Phila Pa 1976) 2012;37:E103-8.

10. Mancuso CA, Duculan R, Stal M, Girardi FP. Patients' expectations of lumbar spine surgery. Eur Spine J 2015;24:2362-9.

11. Brozek JL, Guyatt GH, Schunemann HJ. How a wellgrounded minimal important difference can enhance transparency of labelling claims and improve interpretation of a patient reported outcome measure. Health Qual Life Outcomes 2006;4:69.

12. Parker SL, Adogwa O, Paul AR, et al. Utility of minimum clinically important difference in assessing pain, disability, and health state after transforaminal lumbar interbody fusion for degenerative lumbar spondylolisthesis. J Neurosurg Spine 2011;14:598604.

13. Copay AG, Glassman SD, Subach BR, Berven S, Schuler TC, Carreon LY. Minimum clinically important difference in lumbar spine surgery patients: a choice of methods using the Oswestry Disability Index, Medical Outcomes Study Questionnaire Short Form 36, and pain scales. Spine J 2008;8:968-74.

14. Carragee EJ, Cheng I. Minimum acceptable outcomes after lumbar spinal fusion. Spine J 2010;10:313-20.

15. Parker SL, Godil SS, Shau DN, Mendenhall SK, McGirt MJ. Assessment of the minimum clinically important difference in pain, disability, and quality of life after anterior cervical discectomy and fusion: clinical article. J Neurosurg Spine 2013;18:154-60.

16. Lim JB, Yeo W, Chen JL. Preoperative leg pain score predicts patient satisfaction after transforaminal lumbar interbody fusion surgery. Global Spine J 2018;8:354-8.
17. Macki M, Alvi MA, Kerezoudis P, et al. Predictors of patient dissatisfaction at 1 and 2 years after lumbar surgery. J Neurosurg Spine 2020;32:373-82.

18. Sigmundsson FG, Jonsson B, Stromqvist B. Determinants of patient satisfaction after surgery for central spinal stenosis without concomitant spondylolisthesis: a register study of 5100 patients. Eur Spine J 2017;26:473-80.

19. Massel DH, Mayo BC, Narain AS, et al. Improvements in back and leg pain following a minimally invasive transforaminal lumbar interbody fusion. Int J Spine Surg 2020;14:745-55.

20. Toyone T, Tanaka T, Kato D, Kaneyama R, Otsuka M. Patients' expectations and satisfaction in lumbar spine surgery. Spine (Phila Pa 1976) 2005;30:2689-94.

21. Mannion AF, Junge A, Elfering A, Dvorak J, Porchet F, Grob D. Great expectations: really the novel predictor of outcome after spinal surgery? Spine (Phila Pa 1976) 2009;34:1590-9.

22. Mancuso CA, Duculan R, Stal M, Girardi FP. Patients' expectations of cervical spine surgery. Spine (Phila Pa 1976) 2014;39:1157-62.

23. Asher AL, Kerezoudis P, Mummaneni PV, et al. Defining the minimum clinically important difference for grade I degenerative lumbar spondylolisthesis: insights from the Quality Outcomes Database. Neurosurg Focus 2018;44:E2.

24. Weinstein JN, Lurie JD, Tosteson TD, et al. Surgical vs nonoperative treatment for lumbar disk herniation: the Spine Patient Outcomes Research Trial (SPORT) observational cohort. JAMA 2006;296:2451-9.

25. Parai C, Hagg O, Willers C, Lind B, Brisby H. Characteristics and predicted outcome of patients lost to follow-up after degenerative lumbar spine surgery. Eur Spine J 2020;29:3063-73.

26. Norquist BM, Goldberg BA, Matsen FA 3rd. Challenges in evaluating patients lost to follow-up in clinical studies of rotator cuff tears. J Bone Joint Surg Am 2000;82:838-42.

27. Elkan P, Lagerback T, Moller H, Gerdhem P. Response rate does not affect patient-reported outcome after lumbar discectomy. Eur Spine J 2018;27:153846. 